\title{
The Realization of Multi-touch Electronic Instrument Based on Region Merging Algorithm
}

\author{
Wang Xuechun* and Liu Shenxiao
}

School of Information Engineering, Huanghe Science and Technology College, Zhengzhou, Henan, 450006, P.R. China

\begin{abstract}
This paper has elaborated on the realization process of multi-touch electronic instrument based on region merging algorithm and also made a detailed discussion of the realization principle, object tracking algorithm, software treatment procedure and hardware realization scheme. With the S3C2410A SCM as the core, an intelligent instrument panel that uses multi-touch as the input and color LCD as the output as well as equips itself with the physical-level driver and intermediate software has been developed. This panel has the distinction of high reliability and thus can be widely applied in the electronic instrument with multi-touch technology.
\end{abstract}

Keywords: Human-computer interaction, Multi-touch, Region merging, S3C2410A.

\section{INTRODUCTION}

The touch-screen technology has been commonly used in the instrument, device and other industrial scenarios [1]. For example, most ATM machines have been equipped with the function of touch screen. Many halls in the hospital and library have also been installed with the computer featured by touch-screen technology. There are also a wide range of electronic products, such as cell phone, MP3 and digital camera, which support the touch-screen. However, it should be noted that those existing touch screens have been based on single touch, which means that they can only recognize and support the touch of one finger at a time. In the case of the touch of more than two fingers, the machine will not respond properly. Hence, the multi-touch technology, which is also known as multi-sense, is intended to realize the humancomputer interaction and hardware equipment without the assistance of traditional input equipment such as keyboard and mouse. The multi-touch technology can break down the task into two aspects. Firstly, the signal at multiple points will be gathered. Secondly, the signal of each channel will be perceived, which is also known as gesture recognition. To be more specific, the screen can recognize the click and touch of five fingers at the same time.

The multi-touch technology originated from the multitouch system designed to sense the touch of index finger. The system was invented by Mehta from University of Toronto in 1982 [2]. In the same year, Bell Laboratory also published the first academic article to discuss the technology of touch screen. In 2005, Professor Jefferson Y Han from University of New York led a team to develop the new-type touch screen which could be handled by two hands and even many people at the same time. This technology had greatly pushed forward the commercial operation of multi-touch technology [3]. In 2007, Apple and Microsoft also released the product and plan devoted to multi-touch technology respectively, therefore launching the technology into the mainstream market. This effort has not only laid a solid foundation for the commercial application, but also created great convenience to those common users $[4,5]$. With the aid of multi-touch technology, the user can interact with the machine through multiple touch points. In other words, it has offered a brand-new mode of human-machine interaction, enhanced the experience of the user as well as highly facilitated the operation [6].

Up to today, region merging algorithm has been widely applied in research fields such as digital image segmentation, medical image fusion and statistics. The realization of multitouch technology combined with region merging algorithm can not only optimize the output and recognition but also enhance the reliability of instrument panel.

\section{THE REALIZATION OF MULTI-TOUCH TECH- NOLOGY BASED ON REGION MERGING ALGO- RITHM}

The multi-touch technology has adopted the sensor to perceive the touch of people or object. The sensor will also meet with some problems in the meanwhile. Firstly, if the object touching the screen is relatively big or the touch is applied strongly, the sensor will have a large area to perceive. Secondly, if the object or finger moves along the touch too fast (or the touch has not been applied strongly enough), the scanning system will perceive multiple touching objects rather than the single object within the same scope. Hence, during the course of running the region merging algorithm, we should judge those scanning subjects that are caused by the same object or hand within the same scanning frame. We are also supposed to combine those scanning subjects. Oth- 
erwise, it will be difficult to track or recognize those scanning subjects in movement [7].

The region merging algorithm will be elaborated as follows:

(1) Gather the sampling point from touch screen.The set of sampling point $\mathrm{A}=\{\{\mathrm{Xa} 1, \mathrm{Ya} 1\}, \quad\{\mathrm{Xa} 2, \mathrm{Ya} 2\}$, $\{\mathrm{Xa} 3, \mathrm{Ya} 3\}, \ldots, \quad\{\mathrm{Xan}, \mathrm{Yan}\}\}$ will be gathered from the touch screen and also simultaneously stored by the SDRAM (Synchronous Dynamic Random Access Memory) Fig. (1).

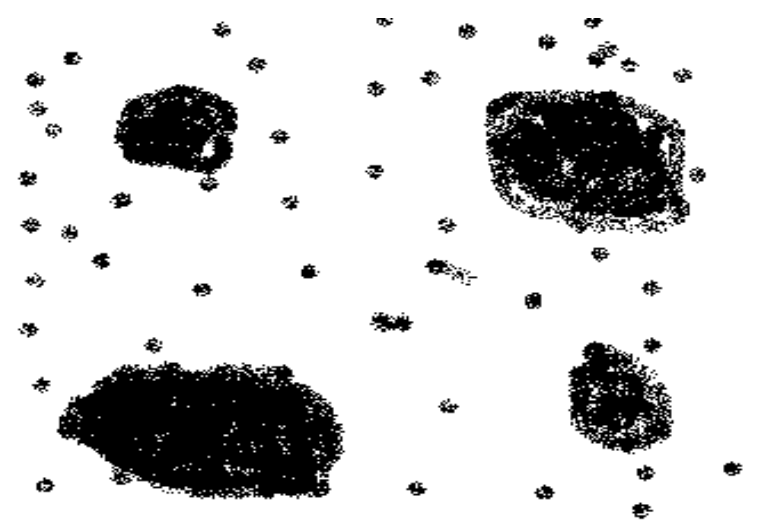

Fig. (1). Touch image of fingers on touch screen.

(2) Digital filtering of gathering points. In this step, the data that has been collected will go through digital filtering, leading to the set $\mathrm{B}$ of touch point, which is $\mathrm{B}=\{\{\mathrm{Xb} 1, \mathrm{Yb} 1\}, \quad\{\mathrm{Xb} 2, \mathrm{Yb} 2\}, \quad\{\mathrm{Xb} 3, \mathrm{Yb} 3\}, \ldots$, $\{\mathrm{Xbm}, \mathrm{Ybm}\}\}$. In this process, a portion of these useless points will be discarded. The parameter of digital filtering will be presented as: exponent number 64, sampling frequency 60 thousand HZ, cut-off frequency 1 thousand $\mathrm{HZ}$ and gain $40 \mathrm{~dB}$.

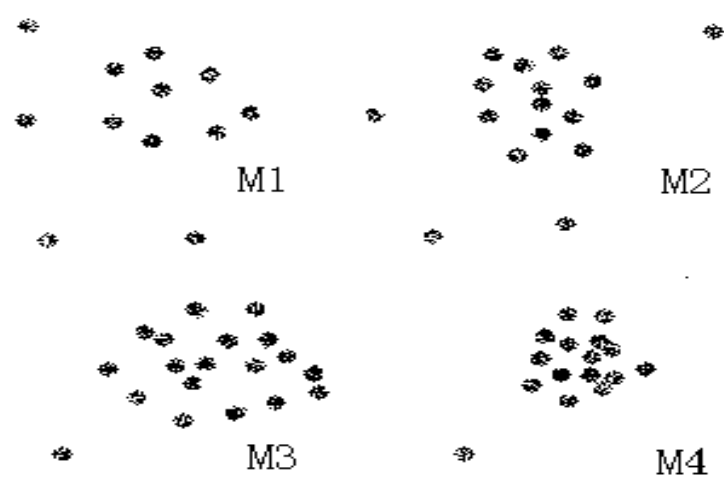

Fig. (2). Digital filtering finishing touch point set B.

(3) Obtain the set of scatted touch point from the outcome of digital filtering. As shown in Fig. (2), four sets of touch screen, namely M1, M2, M3 and M4, have been generated from digital filtering. In this step, the scattered touch points have been identified. According to the $\mathrm{B}(\mathrm{X}, \mathrm{Y})$ of touch point, the relatively concentrated points will form an ellipsoidal area which is closed in many cases. Based on region merging algorithm, the set B will be scanned accordingly to form a sparse matrix in which the element with data will be represented by 1 . Otherwise, it will be represented by 0 . Hence, the area with a concentration of data will be identified, further leading to the sets of M1, M2, M3 and M4.

(4) Identify the sole touch point MC. In the close-ended ellipsoidal area, the central point will be identified. According to the set $\mathrm{M}$ of scattered touch points, the number of those elements in $\mathrm{M}$ will lead to a new matrix. The central area of the matrix will be identified and also seen as the sole touch point MC.

The process of region merging algorithm is presented in Fig. (3).

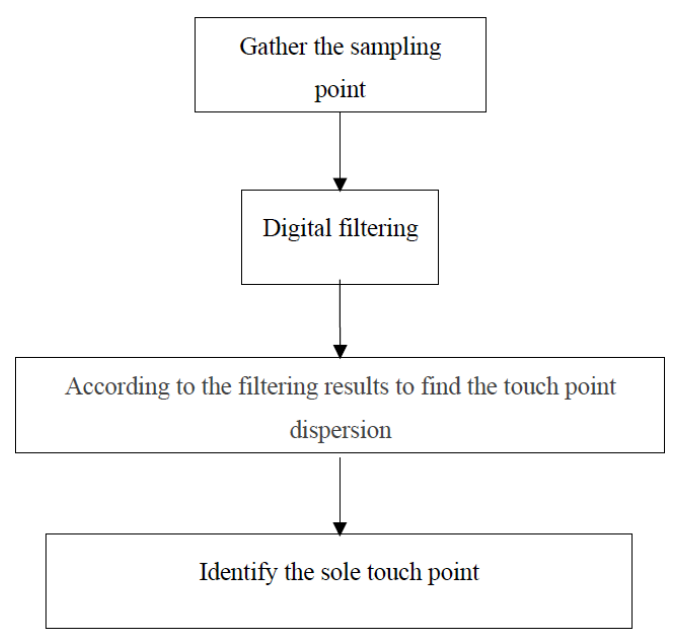

Fig. (3). Region merging algorithm software flow diagram.

\section{THE DESIGN OF SOFTWARE PROCEDURE OF MULTI-TOUCH TECHNOLOGY BASED ON REGION MERGING ALGORITHM}

\subsection{Software Procedure}

Those functions used include:

(1) GetSamplingPoints(): Gather the sampling data from touch screen;

(3) DigitalFiltering(): Digital filtering of sampling point data;

(4) DistributedProcessingTouchPoints(): Scatter the touch point;

(5) FindOnlyTouchPoint(): Search for the sole touch point.

The UML diagram is shown in the Fig. (4).

\subsection{Data Sampling of Touch Point}

(1) The data of touch point that is gathered for the first time will be stored in $\mathrm{A}=\{\mathrm{A} 1, \mathrm{~A} 2, \mathrm{~A} 3, \cdots, \mathrm{Am}\}$. 


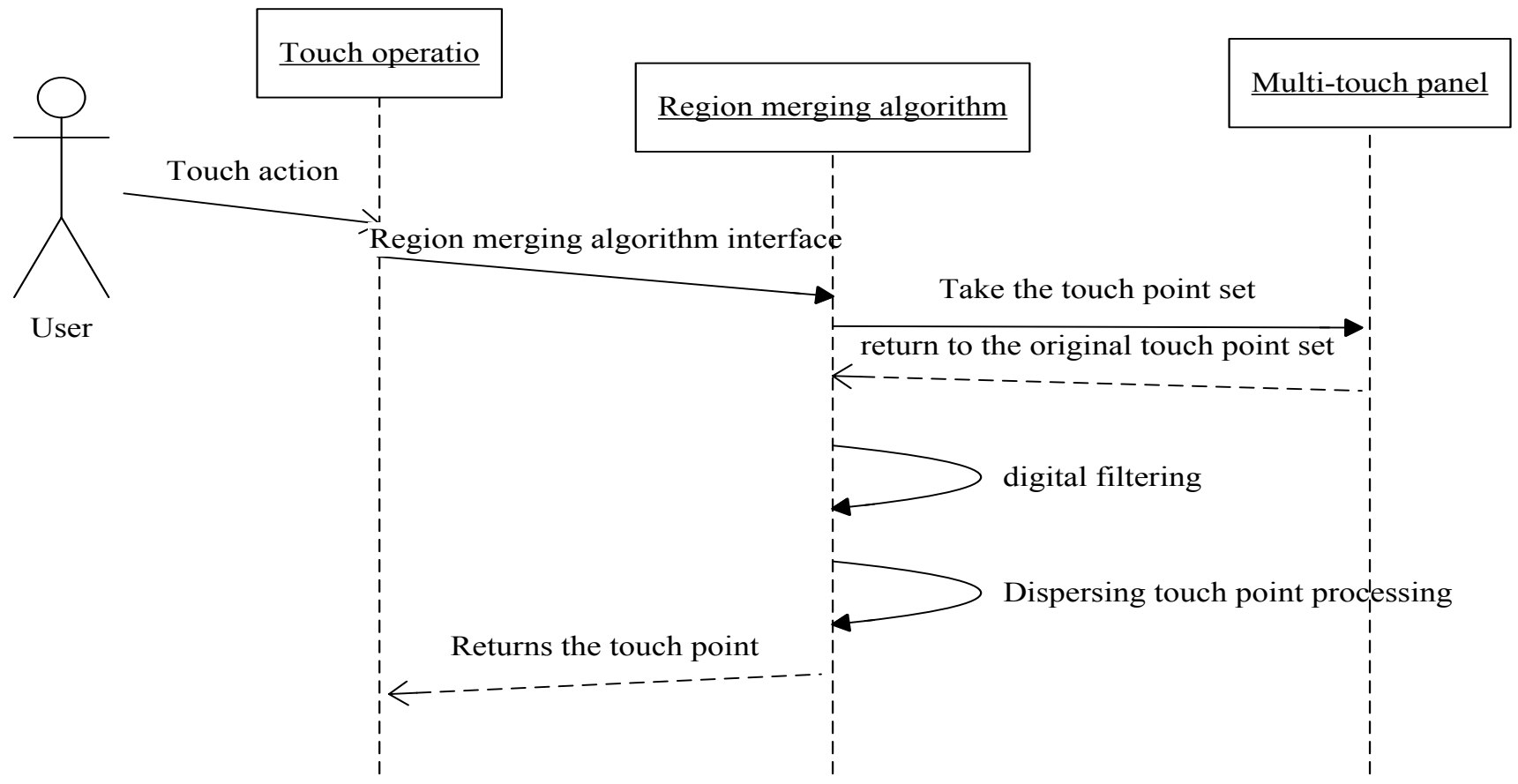

Fig. (4). Region merging algorithm UML diagram.

Based on region merging algorithm, we will combine the sampling data points that are the same or similar to each other to form a new set $\mathrm{C}=\{\mathrm{C} 1, \mathrm{C} 2$, $\mathrm{C} 3, \cdots, \mathrm{Cm}-1\}$. In the meanwhile, the set $\mathrm{A}$ will take the form of coordinate $\{\mathrm{X}, \mathrm{Y}\}$.

(2) The data of touch point that is gathered for the second time will be stored in $\mathrm{B}=\{\mathrm{B} 1, \mathrm{~B} 2, \mathrm{~B} 3, \cdots, \mathrm{Bn}$. According to the step above, we will form a new set $\mathrm{D}=\{\mathrm{D} 1, \mathrm{D} 2, \mathrm{D} 3, \cdots, \mathrm{Dn}-1\}$.

(3) Hence, we have gathered an ellipsoidal area. In the case of fast movement, there will be two separate ellipsoidal areas. If not, the area should be split up from the middle.

(4) As to each sampling point $\mathrm{c}$ in the set C, the length from $\mathrm{c}$ to $\mathrm{d}$ in the set $\mathrm{D}$ will be calculated accordingly. The outcome will be arranged in the ascending order and stored in the array. The elements of long distance in the $\mathrm{E}$ will be also removed.

The data in the E should be optimized. From the first point in E, we will check whether the edge between two corresponding points has been matched well according to various features of the object, such as the stress, area and velocity. It should be made sure that the distance be the shortest. The active contour tracking algorithm and feature tracking algorithm should be also used for judgment. Hence, the match is carried out according to the feature while the sampling point should be also removed accordingly.
(6) Each pair of the data in the set E should be matched. The repeated treatment should be made until all touch points are handled.

(7) From the set $\mathrm{C}$ which is eligible, the most effective data points $\mathrm{Q}=\{\mathrm{Q} 1, \mathrm{Q} 2, \mathrm{Q} 3, \cdots, \mathrm{Qk}\}$ will be gathered, where the value of $K$ ranges from 4 to 10 .

(8) Similarly, the most effective data points $\mathrm{P}=\{\mathrm{P} 1, \mathrm{P} 2$, $\mathrm{P} 3, \cdots, \mathrm{Pk}\}$ will be gathered from the set $\mathrm{D}$ which is eligible, where the value of $\mathrm{K}$ ranges from 4 to 10 .

(9) According to the set $\mathrm{Q}$ and $\mathrm{P}$, the tracking image of touch point will be worked out.

\section{THE DESIGN SCHEME AND TREATMENT PRO- CEDURE OF MULTI-TOUCH HARDWARE}

The design scheme of the multi-touch hardware will be presented in the Fig. (5). With the S3C2410A SCM as the micro-processor and multi-touch technology as the input, this scheme can realize the zooming of screen information. The electronic oscillograph can receive the signal of sine wave. After the transformation from analog signal to digital signal, it will be output in the screen of color LCD. What is more, a series of interface circuits for external equipment such as I2C bus interface, keyboard interface and network card are also available. With the physical-level driver and intermediate software, this panel has the feature of high reliability and thus can be widely applied in the electronic instrument with multi-touch technology. 


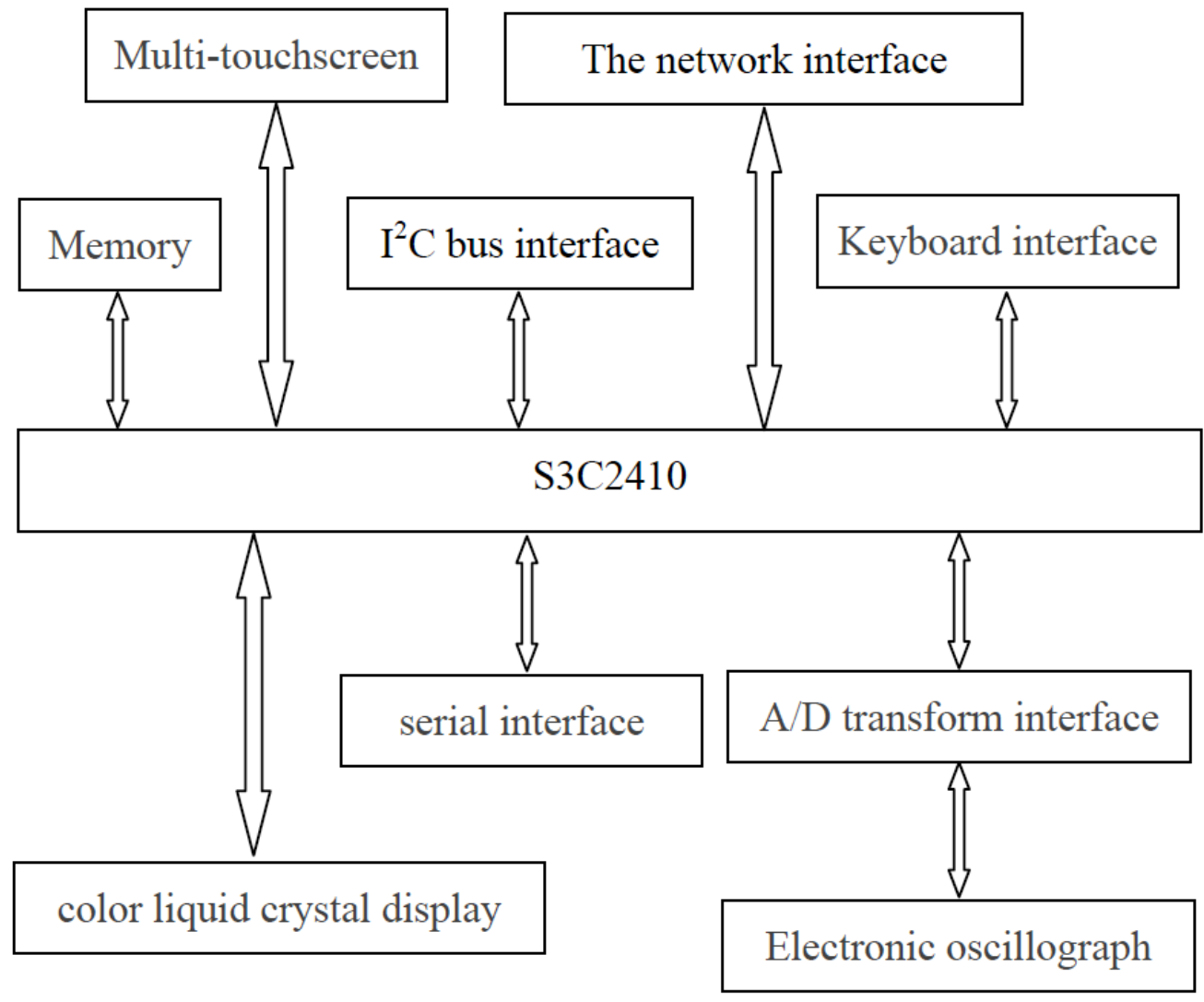

Fig. (5). Multi-touch hardware diagram.

The processing process will include:

(1) Turn on the power supply. The electronic oscillograph is in place to receive the signal of sine wave.

(2) Through $\mathrm{A} / \mathrm{D}$ transformation interface, the signal that has been received will be transformed into the digital signal that can be processed by the machine.

(3) The result will be displayed on the screen of color LED.

(4) The data sensed by the multi-touch technology will be processed and stored.

(5) In a certain interval, the zooming time of the data that is stored will be calculated.

(6) The synchronized zoom of the wave form will be carried out.

\section{CONCLUSION}

This paper has proposed a processor platform with S3C2410A SCM as the core and also realized the multitouch electronic oscillograph based on region merging algorithm. By elaborating on the collection and treatment of touch point as well as the realization of hardware and software, this paper will be also of reference value to the development of multi-touch technology. With the constant development and wide commercial application of multitouch technology, there will be an even brighter prospect for the multi-touch electronic instrument terminal.

\section{CONFLICT OF INTEREST}

The authors confirm that this article content has no conflict of interest.

\section{ACKNOWLEDGEMENTS}

This work was financially supported by Zhengzhou Key Laboratory of embedded System Application Technology, China (Grant No. 121PYFZX177) and Zhengzhou Municipal Natural Science Research Project, China (Grant No. 20141372).

\section{REFERENCES}

[1] F. Zhang, and S. Chen, "Review and prospect of multi-touch interaction mode", Chinese Journal of Ergonomics, vol. 20, pp. 76-78, 2010.

[2] S. Lee, W. Buxton, and K.C. Smith, "A multi-mouch three dimensional touch-sensitive tablet", ACM SIG-CHI Buletin, vol. 16, pp. 21-25, 1985.

[3] J.Y. Han, "Low-cost multi-touch sensing through frustrated total internal reflection", In: Proc.UIST05. ACM, 2005, pp. 115-118. 
[4] Y.H. Xu, S.Q. Li, Y.D. Jia, "A vision-based method for fingerscreen interaction”, Acta Electronica Sinica, vol. 35, pp. 2236-2240, 2007.

[5] M.S. Ou, "Multi-touch technology in handheld devices", Electronic Engineering \& Product World, vol. 15, pp. 70-76, 2008.

[6] C. Forlines, D.Wigdor, C. Shen, and R. Balakrishanan, "Directtouch vs. Mouse input for tabletop displays", In: Proc. CHI 07.ACM, 2007, pp. 647-656.
[7] J.N. Chi, Z.L. Wang, X.Z. Xie, K. Xu, H.L. Fang, Y.Y. Xu, and Y. $\mathrm{Li}$, "A survey of multi-touch human-computer interaction technology", CAAI Transactions on Intelligent Systems, vol. 6, pp. 28-37, 2011.

Received: February 13, 2014

Revised: February 28, 2014

Accepted: October 28, 2014

(C) Xuechun and Shenxiao; Licensee Bentham Open.

This is an open access article licensed under the terms of the Creative Commons Attribution Non-Commercial License (http://creativecommons.org/licenses/by$\mathrm{nc} / 3.0 /$ ) which permits unrestricted, non-commercial use, distribution and reproduction in any medium, provided the work is properly cited. 